\title{
Componentwise fractional programming with application to resource allocation
}

\author{
K. M. MJELDE $\dagger$
}

Keywords: fractional programming, non-linear programming, componentwise programming, resource allocation, optimalization.

A fractional programming problem is considered of the maximization of the ratio of a concave and a convex function, with each variable occurring in a single convex component constraint. A componentwise solution algorithm is given; each component-problem being defined by a weighted difference of the,concave and the convex functions of the given fractional objective function; the relative weight being recalculated after each componentwise optimization.

The proposed algorithm extends those of Dinkelbach and Oettli. An application is given to a fractional resource allocation problem with concave returns and an affine total cost and linear resources. In this problem each componentwise or resourcewise optimization is executed by application of an extension of the algorithm of Luss and Gupta. The results are extended to problems with several time periods connected by a common objective function, but with periodical constraints.

\section{Introduction}

In this paper the following problem, denoted by $R$, is considered:

$$
R: \text { Maximize } z=f(x) / g(x)
$$

subjected to the constraints:

$$
\left.\begin{array}{c}
H_{j}\left(x_{j}\right) \leqslant h_{j} ; j=1, \ldots, J \\
x \geqslant 0
\end{array}\right\}
$$

where $x=\left(x_{j k}\right)$ and $x_{j}=\left(x_{j 1}, \ldots, x_{j K}\right)$ is the $j$ th row of the $J \times K$ matrix $x$. It follows that each variable $x_{j k}$ occurs in exactly one component constraint (1). The functions $f, g$ and $H_{j}$ are differentiable; $f$ is a concave function, $g$ and $H_{j}$ for $j=1, \ldots, J$ are convex functions with $f(x) \geqslant 0$ and $g(x)>0$ for all feasible values of $x$. It is assumed that $H_{j}(0)=0$ and that $h_{j}>0$ for $j=1, \ldots, J$. The maximal objective value of $R$ is denoted by $\bar{z}$ with $\bar{x}$ a corresponding value of $x$.

Since the feasible region of $R$ is compact (and $f$ and $g$ are continuous functions), the problem is solvable by Dinkelbach's (1957) algorithm, which is based on the consideration of an associated problem $R(q)$ defined for any real number $q \geqslant 0$ as follows:

$$
R(q) \text { : Maximize } v(q)=f(x)-q g(x)
$$

subjected to the constraints of $R$. The optimal objective value of $R(q)$ is $\bar{v}(q)$. The steps of the algorithm are as follows:

Received 4 November 1982.

† Det norske Veritas, P.O. Box 300, N-1322 Høvik, Oslo, Norway. 
Step 1

Calculate, for a feasible solution $x^{0}$ of $R$

$$
q_{1}=f\left(x^{0}\right) / g\left(x^{0}\right)
$$

and go to Step 2 with $i=1$.

Step 2

Determine an optimal solution $x$ of the problem $R\left(q_{i}\right)$ and calculate:

$$
q_{i+1}=f\left(x^{i}\right) / g\left(x^{i}\right)
$$

and go to Step 3.

Step 3

Replace $i$ by $(i+1)$ and go to Step 2, if a termination criterion is not satisfied; otherwise terminate the algorithm.

The algorithm produces a non-decreasing sequence $q_{i}=f\left(x^{i-1}\right) / g\left(x^{i-1}\right)$ of objective values of $R$ for $i=1,2, \ldots$ such that

$$
\lim _{i \rightarrow \infty} q_{t}=\bar{z}
$$

For a given $\epsilon>0$ the algorithm may be terminated if

$$
f\left(x^{i}\right)-q_{i} g\left(x^{i}\right) \leqslant \epsilon
$$

-A more satisfactory termination criterion is based on the observation that for each feasible solution $x^{i}$ of $R$, there is an associated upper bound $z_{i}{ }^{*}$ of $\bar{z}$ calculated by the method of Mjelde $(1978,1979)$; if the algorithm is terminated when $z_{i}{ }^{*}-q_{t} \leqslant \epsilon$ it follows that $\bar{z}-q_{i} \leqslant \epsilon$. Additional start and stopping conditions and convergence results were given by Schaible (1976).

The special structure of the problem $R$ considered in this paper is not utilized by the general algorithm of Dinkelbach; in particular, the functions $f$ and $g$ need not be differentiable, nor concave or convex in Dinkelbach's algorithm; and the constraints (1) can be replaced by the requirement that $x$ belongs to any compact subset of $R^{J \times K}$. The purpose of this paper is to propose an algorithm that utilizes the componentwise structure of the problem $R$ with the constraint (1); the proposed algorithm unifies and extends two previously given algorithms; that of Dinkelbach and the componentwise algorithm of Oettli (1974), which was extended by Mjelde (in the press, a) to include certain intermediate improvements between consecutive componentwise optimizations; these algorithms are obtained from the proposed one for respectively $J=1$ and $g(x) \equiv 1$. The new algorithm solves a sequence of problems of the type $R(q)$ previously given, but with a single component constraint (1) for a given value of $j$; the $x_{i}$ for $i \neq j$ being kept at fixed values. The value of $q$ is recalculated after each componentwise optimization by a formula analogous to eqn. (2). It follows that the dimensionality of the problem $R(q)$, to be solved between successive recalculations of $q$ in the componentwise algorithm, is reduced as compared with the general Dinkelbach algorithm. A problem of the allocation of resources to activities is given: each component-optimization being executed by application of an extension of the algorithm of Luss and Gupta (1975), a particular advantage of the proposed 
algorithm when applied to this problem. A concluding section of the paper extends the methods to incorporate several planning periods, indexed by $m \in\{1, \ldots, M\}$, with a separate constraint $H_{j}{ }^{m}\left(x_{j}{ }^{m}\right) \leqslant h_{j}{ }^{m}$ for each $j \in\{1, \ldots, J\}$ and $m=1, \ldots, M$.

\section{The componentwise algorithm}

The steps of the algorithm are as follows:

\section{Step 1}

For a given feasible solution $x^{0}$ of $R$ calculate

$$
q_{1}=f\left(x^{0}\right) / g\left(x^{0}\right)
$$

and go to Step 2 with $n=1$.

\section{Step 2}

For a given non-negative integer $n$ and a given feasible solution $x^{n-1}$ of $R$ determine the resource $j$ such that $j \equiv n(\bmod J)$, which means that $j=n-k J$ for some integer $k$, and determine an optimal solution $x_{j}=x_{j}^{n} \geqslant 0$ of the problem $R_{n}\left(q_{n}\right)$ defined as follows:

$$
R_{n}\left(q_{n}\right): \text { Maximize } z=f(x)-q_{n} g(x)
$$

subjected to the constraint:

$$
H_{j}\left(x_{j}\right) \leqslant h_{j} ; j \equiv n(\bmod J)
$$

and the requirements that $x_{i}=x_{i}{ }^{n-1}$ are given for all $i \neq j$.

An optimal solution of $R_{n}\left(q_{n}\right)$ is denoted by $x^{n}$, where the rows $x_{i}^{n}$ of $x^{n}$ are given for $i=1, \ldots, J$ from:

$$
x_{i}^{n}= \begin{cases}x_{j}^{n} & \text { if } i=j \\ x_{i}^{n-1} & \text { if } i \neq j\end{cases}
$$

Calculate

$$
q_{n+1}=f\left(x^{n}\right) / g\left(x^{n}\right)
$$

and go to Step 3 .

\section{Step 3}

Replace $n$ by $(n+1)$ and go to Step 2 or terminate the algorithm according to a given criterion.

The previously given termination criteria of the Dinkelbach algorithm can be applied to the new algorithm as well.

\section{Lemma I}

For the componentwise algorithm it follows, for $n=1,2, \ldots$, that :

$$
\begin{aligned}
& q_{n} \leqslant \bar{z} \\
& q_{n} \leqslant q_{n+1}
\end{aligned}
$$


Proof

Equations (3) and (4) demand that $q_{n} \leqslant \bar{z}$. The definition of $x^{n}$ in Step 2 and the definition of $q_{n}$ show that:

$$
f\left(x^{n}\right)-q_{n} g\left(x^{n}\right) \geqslant f\left(x^{n-1}\right)-q_{n} g\left(x^{n-1}\right)=0
$$

and consequently that

$$
q_{n} \leqslant f\left(x^{n}\right) / g\left(x^{n}\right)
$$

which, in combination with eqn. (4), requires that:

$$
q_{n} \leqslant q_{n+1} \text {, q.e.d. }
$$

An extension of a proof of Oettli (1974) gives the following:

\section{Theorem}

For the componentwise algorithm it follows that:

$$
\lim _{n \rightarrow \infty} q_{n}=\bar{z}
$$

\section{Proof}

For notational convenience, for a feasible solution $x$ of $R$, define the transponse of $x$ as follows:

$$
\xi=x^{T}=\left(\xi_{1}, \ldots, \xi_{J}\right)
$$

where

$$
\xi_{j}=x_{j}^{T}
$$

The following notations are introduced:

$$
\begin{aligned}
\xi_{j-} & =\left(\xi_{1}, \ldots, \xi_{j-1}\right) \text { for } j \geqslant 2 \\
\xi_{j+} & =\left(\xi_{j+1}, \ldots, \xi_{J}\right) \text { for } j \leqslant J-1 \\
\xi & =\left(\xi_{1}, \xi_{1+}\right)=\left(\xi_{J-}, \xi_{J}\right) \\
\xi & =\left(\xi_{j-}, \xi_{j}, \xi_{j+}\right) \text { for } 2 \leqslant j \leqslant J-1
\end{aligned}
$$

It follows from the definition of the algorithm and $n=k J+j$ that

$$
\xi^{n}=\left(x^{n}\right)^{T}=\left(\xi_{j-}{ }^{(k+1) J}, \xi_{j}^{(k+1) J}, \xi_{j+}{ }^{k J}\right)
$$

If the objective value of the problem $R$ corresponding to $x^{n}=\left(\xi^{n}\right)^{T}$ is denoted by $z\left(\xi^{n}\right)$ it follows from the definitions (3) and (4) and the property (6) of the Lemma that:

$$
\begin{aligned}
z\left(\xi^{k J}\right) & \leqslant z\left(\xi_{j-}{ }^{(k+1) J}, \xi_{j}{ }^{k J},{\xi_{j+}}^{k J}\right) \\
& \leqslant z\left(\xi_{j-}{ }^{(k+1) J}, \xi_{j}{ }^{(k+1) J}, \xi_{j+}{ }^{k J}\right) \\
& \leqslant z\left(\xi^{(k+1) J}\right)
\end{aligned}
$$

Since the feasible region of $R$ is closed and bounded, consideration of the sequence $\left\{\xi^{k J}, \xi^{(k+1) J}\right\}$ shows that it contains a convergent subsequence, with the limit denoted by $\{\bar{\theta}, \bar{\xi}\}$. Noting that, by the monotonicity of the sequence $\left\{q_{n}\right\}$, it follows that $z(\ddot{\theta})=z(\bar{\xi})$, taking limits in eqn. (7) gives:

$$
z(\bar{\theta})=z\left(\xi_{j-}, \bar{\theta}_{j}, \bar{\theta}_{j+}\right)=z\left(\xi_{j-}, \bar{\xi}_{j}, \tilde{\theta}_{j+}\right)=z(\bar{\xi})
$$


The properties (5) and (6) of Lemma 1 demand that the sequence $\left\{q_{n}\right\}$ is convergent:

$$
\lim _{n \rightarrow \infty} q_{n}=q^{*}
$$

For $\xi=x^{T}$ consider the function:

$$
z^{*}(\xi)=f(x)-q^{*} g(x)
$$

and the problem $R^{*}$ of the maximization of $z^{*}(\xi)$ for all feasible solutions $x$ of $R$. Since the definition (4) of $q_{n+1}$ demands that

it follows that

$$
q^{*}=z(\bar{\theta})=z(\bar{\xi})
$$

$$
z^{*}(\bar{\theta})=z^{*}(\bar{\xi})=0
$$

Equations (8) and (9) imply that:

$$
z^{*}\left(\bar{\xi}_{j-}, \bar{\theta}_{j}, \bar{\theta}_{j+}\right)=z^{*}\left(\xi_{j-}, \bar{\xi}_{j}, \bar{\theta}_{j+}\right)
$$

Since, for $n=k J+j$, the vector $\xi_{j}{ }^{(k+1) J}$ is an optimal solution of $R_{n}\left(q_{n}\right)$ with $\xi_{j-}{ }^{(k+1) J}$ and $\xi_{j+}{ }^{k J}$ given, the continuity of the functions $f$ and $g$, eqn. (11) and $\lim _{n \rightarrow \infty} q_{n}=q^{*}$
imply the validity of the following property:

$$
\bar{\xi}_{j} \text { and } \bar{\theta}_{j} \text { are optimal solutions of } R^{*} \text { with } \xi_{j-} \text { and } \bar{\theta}_{j+} \text { given }
$$

The case $j=1$ shows that $\bar{\xi}_{1}$ is an optimal solution of $R^{*}$ with $\bar{\theta}_{1+}$ given, which implies that $\left(\xi_{1}, \bar{\theta}_{2}\right)$ is an optimal solution of $R^{*}$ with $\bar{\theta}_{2+}$ given, because the above property (12) requires that $\bar{\theta}_{2}$ is optimal with $\bar{\xi}_{1}$ and $\bar{\theta}_{2+}$ given; and the componentwise optimality of $\left(\xi_{1}, \bar{\theta}_{2}\right)$ with $\bar{\theta}_{2+}$ given implies optimality (since $z^{*}$ is a concave and differentiable function of $x$ ). It follows from eqn. (11) that $\left(\xi_{1}, \xi_{2}\right)$ is optimal with $\bar{\theta}_{2+}$ given, and by induction that $\xi=\left(\xi_{1}, \xi_{2}, \ldots, \bar{\xi}_{J}\right)$ is an optimal solution of $R^{*}$.

The implication is that, for any feasible solution $x$ of $R$ :

$$
f(x)-q^{*} g(x) \leqslant z^{*}(\bar{\xi})
$$

and since $z^{*}(\xi)=0$ by eqn. (10) it follows that:

$$
f(x) / g(x) \leqslant q^{*}
$$

and consequently that $q^{*}=\bar{z}$, q.e.d.

\section{Application to a resource allocation problem}

The following problem, denoted by $R A$, of the allocation of resources $j \in\{1, \ldots, J\}$ to activities $k \in\{1, \ldots, K\}$ was considered by Mjelde (1978):

$$
R A \text { : Maximize } z=\sum_{k=1}^{K} r_{k}\left(\sum_{j=1}^{J} \alpha_{j k} x_{j k}\right) /\left(\beta_{0}+\sum_{i=1}^{J} \sum_{k=1}^{K} \beta_{j k} x_{j k}\right)
$$

subjected to the constraints:

$$
\begin{aligned}
\sum_{k=1}^{K} x_{j k} \leqslant h_{j} ; j=1, \ldots, J \\
x_{j k} \geqslant 0 ; j=1, \ldots, J ; \quad k=1, \ldots, K
\end{aligned}
$$

where $\alpha_{j k}$ and $\beta_{j k}$ are respectively the effectiveness and the cost of allocating a unit of resource $j$ to activity $k ; \beta_{0}$ is a fixed cost; $x_{j k}$ the quantity of resource $j$ allocated to 
activity $k ; h_{j}$ the available amount of resource $j$; and $r_{k}$ is a concave, strictly increasing and differentiable function with $r_{k}(0)=0$, describing the return from activity $k$ due to given allocations. It is assumed that $\alpha_{j k} \geqslant 0$ and $\beta_{j k} \geqslant 0$ for $j=1, \ldots, J$ and $k=1, \ldots, K$; that $h_{j}>0$ for $j=1, \ldots, J$ and $\beta_{0}>0$.

The problem $R A$ can be solved by application of the componentwise algorithm of this paper. The objective function of a problem $R_{n}\left(q_{n}\right)$ is maximized subject to the constraints $(13)$ and $(14)$ for a given $j \equiv n(\bmod J)$.

An efficient solution algorithm for the case $q_{n}=0$ was given by Luss and Gupta (1975). This algorithm can be extended to the problem $R_{n}\left(q_{n}\right)$ with $q_{n}>0$, as noted by Mjelde (in the press, b). The extension is as follows:

Define the marginal returns:

$$
\begin{aligned}
\eta_{j k}{ }^{0} & =\alpha_{j k} r_{k}^{\prime}\left(\sum_{i \neq j} \alpha_{i k} x_{i k}{ }^{n-1}\right)-q_{n} \beta_{j k} ; \quad k=1, \ldots, K \\
\eta_{j, K+1}{ }^{0} & =0
\end{aligned}
$$

and assume, without loss of generality, that the activities $k \in\{1, \ldots, K\}$ have been ordered such that

If, for

$$
\eta_{j k}{ }^{0} \geqslant \eta_{j, k+1}{ }^{0} ; \quad k=1, \ldots, K
$$

$$
\eta_{j}{ }^{0}=\operatorname{Max}_{k} \eta_{j k}^{0}
$$

it follows that $\eta_{j}{ }^{0} \leqslant 0$, the Kuhn-Tucker conditions of $R_{n}\left(q_{n}\right)$ show that $\left(x_{j k}\right)=(0)$ is an optimal solution. If $\eta_{j}{ }^{0}>0$, define an integer $L_{j}{ }^{\circ} \in\{1, \ldots, K\}$ such that:

$$
\eta_{j k}{ }^{0}>0 \text { if and only if } k \in\left\{1, \ldots, L_{j}{ }^{0}\right\}
$$

and determine $x_{j k}{ }^{0}$ for $k=1, \ldots, L_{j}{ }^{0}$ from the equations:

$$
\alpha_{j k} r_{k}^{\prime}\left(\alpha_{j k} x_{j k}{ }^{0}+\sum_{i \neq j} \alpha_{i k} x_{i k}^{n-1}\right)=q_{n} \beta_{j k}
$$

If

$$
\sum_{k=1}^{L, 0} x_{j k}^{0}<h_{j}
$$

the optimal solution of $R_{n}\left(q_{n}\right)$ is given by $x_{j k}=x_{j k}^{0}$ for $k=1, \ldots, L_{j}^{0}$ and $x_{j k}=0$ if $k=L_{j}{ }^{0}+1, \ldots, K$, since the Kuhn-Tucker conditions of $R_{n}\left(q_{n}\right)$ become satisfied. If eqn. (16) is not satisfied the original Luss-Gupta algorithm applies directly.

Equation (15) can be solved explicitly for a class of return functions $r_{k}$, including the functions:

$$
\begin{aligned}
& r_{k}(\xi)=a_{k}\left[1-\exp \left(-b_{k} \xi\right)\right], \quad \xi \geqslant 0, \quad a_{k}>0, \quad b_{k}>0 \\
& r_{k}(\xi)=s_{k} \xi-m_{k} \xi^{2} ; \quad 0 \leqslant \xi \leqslant s_{k} / 2 m_{k}, \quad s_{k}>0, \quad m_{k}>0 \\
& r_{k}(\xi)=s_{k} \ln \left(1+m_{k} \xi\right), \quad \xi \geqslant 0, \quad s_{k}>0, \quad m_{k}>0 \\
& r_{k}(\xi)=s_{k}\left(\xi+c_{k}\right) /\left(\xi+m_{k}\right), \quad \xi \geqslant 0, \quad m_{k}>c_{k}>0
\end{aligned}
$$

Luss and Gupta demonstrated that their algorithm is particularly efficient for these functions, due to the explicit invertibility of certain equations to be solved in the algorithm. Otherwise these equations may be solved numerically by the application of the algorithm of Einbu (1981). 


\section{Concluding remarks}

A problem identical to $R$, but with several planning periods can be reduced to the type $R$ by a transformation of variables: such problems include time dependent or multi-period resource allocation problems. Explicitly, the problem $R N$ is given by:

$$
R N \text { : Maximize } z=f\left(x^{1}, \ldots, x^{M}\right) / g\left(x^{1}, \ldots, x^{M}\right)
$$

subject to the constraints:

$$
\begin{aligned}
& H_{j}{ }^{m}\left(x_{j}{ }^{m}\right) \leqslant h_{j}^{m} ; \quad j=1, \ldots, J, \quad m=1, \ldots, M \\
& x_{j}{ }^{m} \geqslant 0 ; \quad j=1, \ldots, J ; \quad m=1, \ldots, M
\end{aligned}
$$

where $x_{j}{ }^{m}=\left(x_{j 1}{ }^{m}, \ldots, x_{j K}{ }^{m}\right)$ is the $j$ th row of $x^{m}$ for $m=1, \ldots, M$.

A lexicographical transformation $t(j, m)$ of the indices $(j, m)$ defined by:

$$
t(j, m)=(m-1) J+j
$$

gives a problem of the type $R$ considered in this paper. Explicitly, define $x_{t}^{\prime}=x_{j}{ }_{j}$, where $x_{t}^{\prime}$ is the $t$ th row of the matrix $x^{\prime}=\left(x_{t k}^{\prime}\right)$ with $J . M$ rows and $K$ columns, and introduce the following notations:

$$
H_{t}^{\prime}\left(x_{t}^{\prime}\right)=H_{j}^{m}\left(x_{j}^{m}\right)
$$

$h_{t}^{\prime}=h_{j}{ }^{m} ; f^{\prime}\left(x^{\prime}\right)=f\left(x^{1}, \ldots, x^{M}\right)$; and $g^{\prime}\left(x^{\prime}\right)=g\left(x^{1}, \ldots, x^{M}\right)$.

\section{REFERENCES}

Dinkelbach, W. (1967). On nonlinear fractional programming. Management Science, 13, 492-498.

EinBu, J. M. (1982). Extension of the Luss-Gupta resource allocation algorithm by means of first order approximation techniques. Operations Research, 29, 621-626.

Luss, H., and GUPTA, S. (1975). Allocation of effort resources among competing activities. Operations Research, 23, 360-366.

MJELDE, K. M. (1978). Allocation of resources according to a fractional objective. European Journal of Operational Research, 2, 116-124.

MJELDE, K. M. (1979). Convex-concave fractional programming-evaluation of solutions and optimality conditions. $B I T, 19,270-271$.

MJELDE, K. M. (in the press). (a). Componentwise optimization with intermediate improvements. Zeitschrift für Angewandte Mathematik und Mechanik; (b). Methods of the Allocation of Limited Resources (John Wiley \& Sons Limited, May 1983).

OettLI, W. (1974). Einzelschritt-Verfahren zu Lösung Konvexer und Dual-Konvexer Minimierungsprobleme. Zeitschrift für Angewandte Mathematik und Mechanik, 54, 343-351.

SCHAibLE, S. (1976). Fractional programming I, duality. Management Science, 22, 858-867. 